\title{
Optimization of Arrangement of LED on the PCB for High Power LED Module
}

\author{
Ju Yong Cho, Ho Seob Kim, Won Kweon Jang
}

\begin{abstract}
LED operating under high-temperature condition badly affects reliability. To reduce junction temperature of $L E D$ is crucial. In this paper, luminous intensity and photo conversion efficient with respect to electrical power are discussed. Moreover, three arrangements for LED module are suggested, and design parameters are discussed in terms of the number of LEDs and distance between each LED. In order to evaluate thermal performance of designed the module, computer simulation was conducted. Distance between each LED is selected by 7.6, 9.6, and $13.3 \mathrm{~mm}$ for 80,128 , and $240 \mathrm{LEDs}$, respectively and unit heat flux is calculated to be $0.47 \mathrm{~W} / \mathrm{mm}^{2}, 0.29 \mathrm{~W} / \mathrm{mm}^{2}, 0.16 \mathrm{~W} / \mathrm{mm}^{2}$ for 80 , 128, and 240 LEDs, respectively. In this case, Maximum temperature on the $P C B$ was $67.8^{\circ} \mathrm{C}, 62.5^{\circ} \mathrm{C}$, and $57.1^{\circ} \mathrm{C}$ for 80 , 128, and 240 LEDs, respectively. The Maximum temperature and unit heat flux was reduced by $15.7 \%$ and $66 \%$, respectively, when the number of LEDs are increased by three times. We found that the temperature between LEDs can be reduced if unit heat flux can be reduced.
\end{abstract}

Keywords : Thermal resistance, Thermal degradation, High power LEDs, Heat transfer, Heat flux.

\section{INTRODUCTION}

LED is comparatively more energy efficient, environment friendly, longer life, lighter mass and smaller volume than the conventional luminaries of fluorescent lamp and incandescent lamp. Though the photo conversion efficiency of the LED of $15-25 \%$ is about twice of $10 \%$ of fluorescent lamp, the practical efficiency is much higher considering the spectrum matching to useful illumination. Therefore, LED has become a strong alternative replacing the artificial luminaries in various fields of industry [1].

Recently, LED has continuously widened its application as the power of single LED grows higher than watt level, however, the junction temperature has also increased with the power of single LED growing. Thermal problem is still existing problem again due to the degradation of luminous intensity and lifetime as well as wavelength shift [1].

To avoid many disadvantages caused by excessive heat generation and insufficient heat extraction from $p-n$ junction, the temperature of LED should be maintained under a certain temperature that guarantee the normal operation as designed and expected. Normally, brighter illumination requires more number of small power LED or small number of LED with higher power.

Revised Manuscript Received on December 30, 2019.

* Correspondence Author

Ju Yong Cho, Ph.D Candidate, Optoelectronics, Hanseo university, Seosan, South Korea.

Ho Seob Kim, Professor, Department of Nanoscience, Sunmoon University, Asan, South Korea.

Won Kweon Jang, Professor, Department of Aeronautic Electricity, Hanseo University, South Korea.

(C) The Authors. Published by Blue Eyes Intelligence Engineering and Sciences Publication (BEIESP). This is an open access article under the CC BY-NC-ND license (http://creativecommons.org/licenses/by-nc-nd/4.0/)
If large number of LEDs are attached to PCB(Printed circuit board) to get a certain illumination, the each LED requires low electrical energy compared to small number of high-power LEDs. In this case, though the heat generation from each LED can be significantly reduced, it is not sure the temperature of each LED to be maintained low because the distance between LEDs is closer due to large number of LED. Furthermore, the heat dissipation of LEDs is inefficient because the dielectric layers of the LED and the PCB have low thermal conductivity of $0.3-3.0 \mathrm{~W} / \mathrm{mK}[2]-[4]$. МСPCB, metallic layer of high thermal conductivity attached to the PCB, is now widely used to increase thermal conductivity of the PCB [5]. Another method to reduce the junction temperature is to find the optimum arrangement of proper number of LEDs on the PCB. In this paper, we calculated parameters of heat flux and thermal resistance to find the most effective LED lighting system in three arrangements of LEDs on the PCB. In each arrangement of the LEDs the unit heat flux and temperature distribution are also discussed.

\section{THERMAL RESISTANCE ANALYSIS}

In the medium heat transfers from high temperature to low temperature region. Heat flux in the medium depends on thermal conductivity, area, length, and temperature difference as follows:

$$
Q=k \frac{T_{1}=T_{2}}{L}
$$

Where $Q$ is heat flux, $T_{1}$ is highest temperature, $T_{2}$ is lowest temperature, $k$ is thermal conductivity, and $L$ is length of medium. If heat is exchanged between surface of medium and fluid that meets the surface, heat flux can be given as [6]-[8]:

$$
Q=h T_{n}=T_{\text {outer }}
$$

Where, $T_{n}$ is temperature at $n$th point on the surface, $T_{\text {outer }}$ is fluid temperature, $h$ is convection coefficient and $n$ is integer.

Thermal resistance is used to evaluate thermal performance of electronic devices and the resistance is defined as follows [6]-[8]:

$$
R=\frac{T_{1}-T_{2}}{Q}
$$

Where $R$ is the thermal resistance. Fig. 1 shows the many LEDs attached on the PCB and its equivalent thermal circuit. If the number of LED on the PCB is increased, each LED requires less electrical energy when $Q_{\text {tot }}$ is assumed to be constant. In order to reduce junction temperature, the arrangement and the number of LEDs on the PCB are crucial. 
In this case, total thermal resistance and heat generation can be written as follows:

$$
\begin{aligned}
& Q_{\text {tot }}=Q_{1}+Q_{2}+Q_{3}+\cdots+Q_{n} \\
& \approx\left(T_{1}-T_{2}\right) \frac{1}{R_{1}+R_{2}+R_{3}+\cdots+R_{4}}=\frac{\left(T_{1}-T_{2}\right)}{R_{\text {tot }}}
\end{aligned}
$$

Where $Q_{\text {tot }}$ is total heat, $Q_{n}$ is individual heat which produced from the LED, $R_{\text {tot }}$ is total thermal resistance, $R_{n}$ is thermal resistance of individual LED, and $n$ is integer.

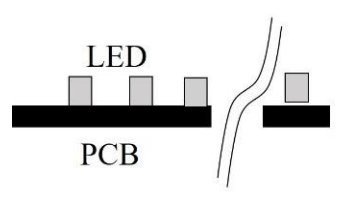

(a)

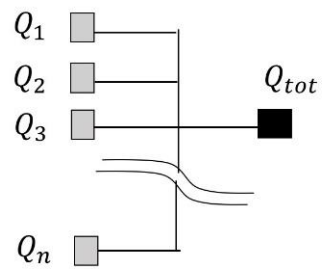

(b)
Fig 1. Schematic diagram. (a) LEDs attached on the PCB, (b) thermal equivalent circuit for LEDs

Heat flux of LED can significantly be lowered in case of the large number of LED on the PCB. Unit heat flux for each LED can be calculated as.

$$
Q=\frac{Q_{\text {tot }} \times \eta}{n \times a}
$$

Where $\eta$ is thermal conversion efficiency, $n$ is the number of LED, $a$ is the surface area of single LED.

\section{RESULT AND DISCUSSION}

We investigated photo conversion efficiency and luminous intensity of LED module with respect to the electrical input power. The module of 12 LED was placed inside integrating sphere, and the electrical power applied to the module with adjusting the electrical input power from $1.3 \mathrm{~W}$ to $5.7 \mathrm{~W}$ to measure the luminous intensity and the photo conversion efficiency. Single LED consumes $100 \mathrm{~mW}$ and $480 \mathrm{~mW}$ when $1.3 \mathrm{~W}$ and $5.7 \mathrm{~W}$ applied to the module, respectively. Fig. 2 shows the luminous intensity and photo conversion efficiency versus the electrical input power applied to the LED. The luminous intensity was 328lm at $1.3 \mathrm{~W}$ and it was increased to $1100 \mathrm{~lm}$ at $5.7 \mathrm{~W}$. Photo conversion efficiency was $246.5 \mathrm{~lm} / \mathrm{W}$ at $1.3 \mathrm{~W}$ and it was decreased to $192.6 \mathrm{~lm} / \mathrm{W}$ at $5.7 \mathrm{~W}$. Photo conversion efficiency was decreased by $21.8 \%$ when the electrical power is increased from $1.3 \mathrm{~W}$ to $5.7 \mathrm{~W}$. According to this result, the temperature of each LED should be kept as low as possible.

Computer simulation was performed to evaluate the thermal performance of LEDs. The distance between each LED were 7.6, 9.6, and 13.3mm when the number of LEDs were 240, 128 and 80, respectively. Thermal conversion efficiency and total electrical input power are assumed as $75 \%$ and $100 \mathrm{~W}$. The surface area of single LED is about $2.0 \mathrm{~mm}^{2}$, and the calculated unit heat flux were $0.47 \mathrm{~W} / \mathrm{mm}^{2}$, $0.29 \mathrm{~W} / \mathrm{mm}^{2}$, $0.16 \mathrm{~W} / \mathrm{mm}^{2}$ for 80,128 , and $240 \mathrm{LEDs}$, respectively. When the number of LED is increased by three times, the unit heat flux is decreased by $65.9 \%$. Fig. 3 shows the simulated model for 240 LEDs.

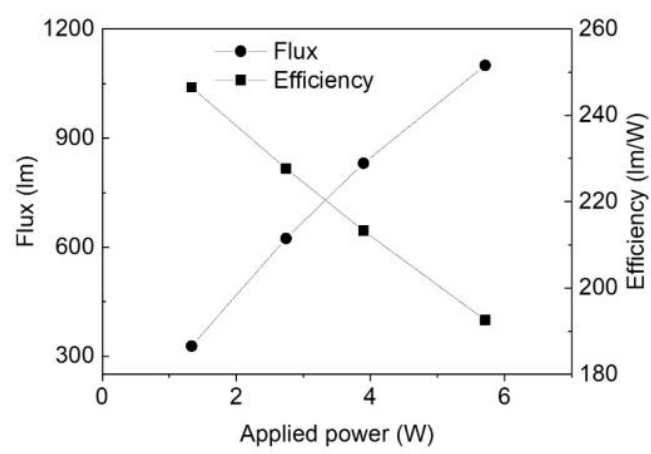

Fig. 2. Luminous intensity and photo conversion efficiency with respect to applied electrical power.

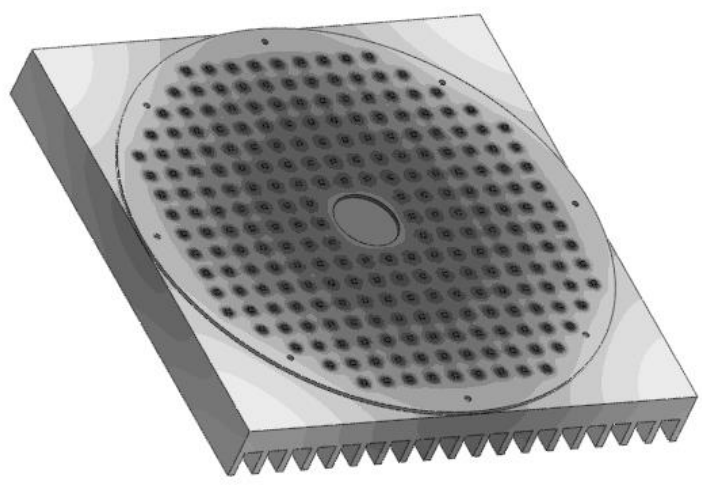

Fig. 3. Simulation model

The diameter of MCPCB is $300 \mathrm{~mm}$ and it is attached to the aluminum heatsink. The width, length and thickness of the heat sink are $300 \mathrm{~mm}, 300 \mathrm{~mm}$ and $18 \mathrm{~mm}$, respectively. The thermal conductivity of metal core PCB is $8.5 \mathrm{~W} / \mathrm{mK}$ $[9,10]$. LED is assumed as GaN. Thermal conductivity of LED is $130 \mathrm{~W} / \mathrm{mK}$ and thermal conductivity of heatsink is $204 \mathrm{~W} / \mathrm{mK}[9,10]$. Convection coefficient, $\mathrm{h}$ is normally from $5 \times 10^{-6} \mathrm{~W} / \mathrm{mm} 2$ to $30 \times 10^{-6} \mathrm{~W} / \mathrm{mm}^{2}$. In this simulation, the coefficient is assumed to be $30 \times 10^{-6} \mathrm{~W} / \mathrm{mm}^{2}$ [10]. Fig. 4 shows the simulated thermal image. Darker region represent higher temperature. The temperature of the center position, indicated 1 in fig. 4 located along the horizontal line, was used from comparison. The highest temperatures at 1 position were $67.8^{\circ} \mathrm{C}, 62.5^{\circ} \mathrm{C}$, and $57.1^{\circ} \mathrm{C}$ for modules of 80,128 , and 240 LEDs, respectively. The lowest temperatures were $49.7^{\circ} \mathrm{C}, 50.6^{\circ} \mathrm{C}, 50.3^{\circ} \mathrm{C}$ for modules of 80,128 , and 240 LEDs, respectively. Those temperature distributions are shown in fig. 5. The module of 80 LEDs shows the lowest temperature distribution. The thermal conductivity of PCB is quite low compared to aluminum heat sink. The heat surely transferred to the heat sink rather than the surface direction of MCPCB. Longer distance between each LED showed the less heat transfer along the PCB surface. Therefore, the module of 80 LEDs shows the lowest temperature at the position between LEDs on the MCPCB in three arrangements. However, when we compared the modules of 128 LEDs and 240 LEDs, the temperature at the position between LEDs in module of $240 \mathrm{LEDs}$ shows $0.3^{\circ} \mathrm{C}$ lower than that of the 128 LEDs module because the unit heat flux of 240 LEDs module is significantly lower than that of 128 LEDs module.

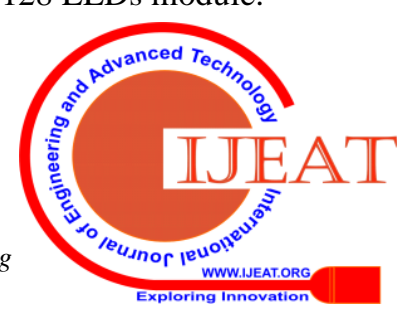




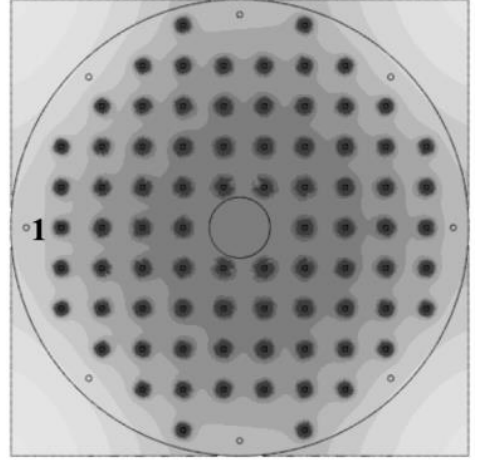

(a)

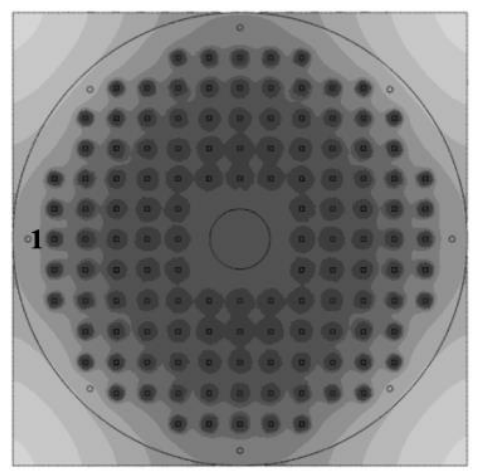

(b)

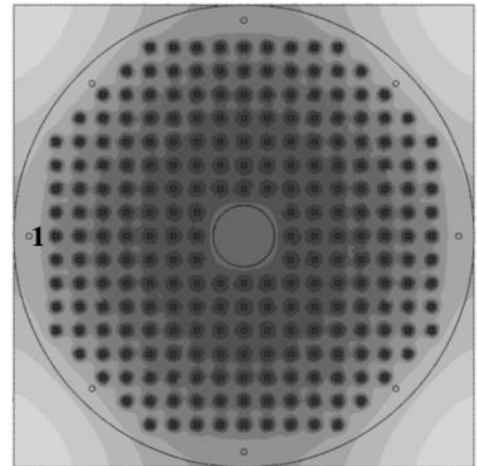

(c)

Fig. 4. Simulated thermal image. (a) 80LEDs, (b) 128LEDs, (c) 240LEDs

Fig. 5 shows that the temperature of LED p-n junction depends on the unit heat flux. The temperatures of the spike top represent the $\mathrm{p}-\mathrm{n}$ junction temperature, and the temperatures of bottom background are for the between LEDs.

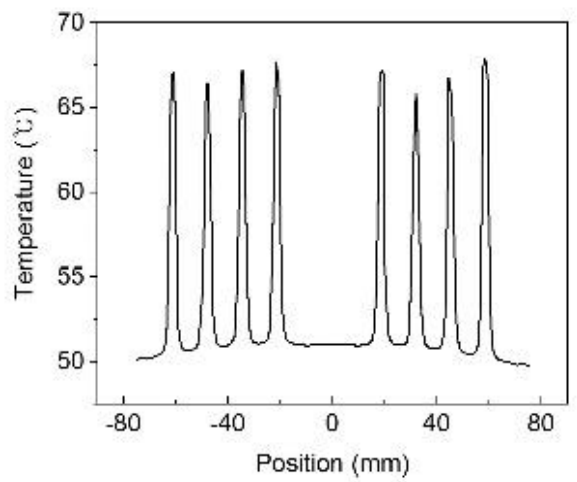

(a)

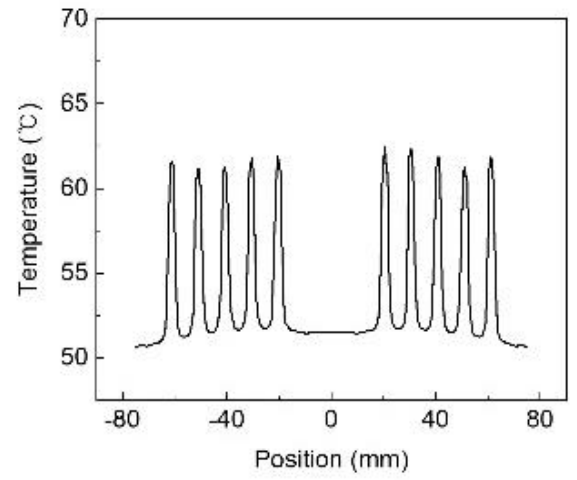

(b)

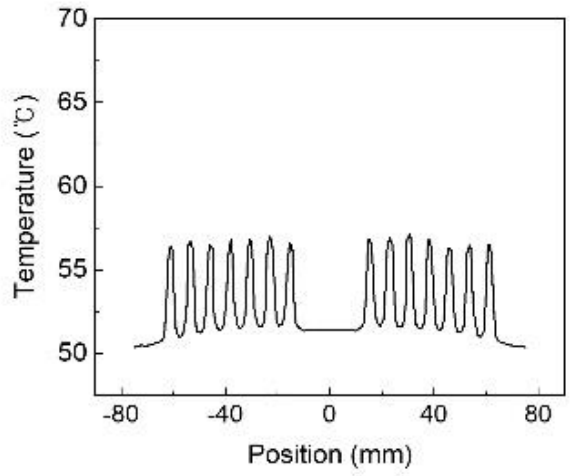

(c)

Fig. 5. Temperature distribution along horizontal line at the center, indicated as 1 in fig. 4 (a) 80LEDs, (b) 128LEDs, (c) 240LEDs

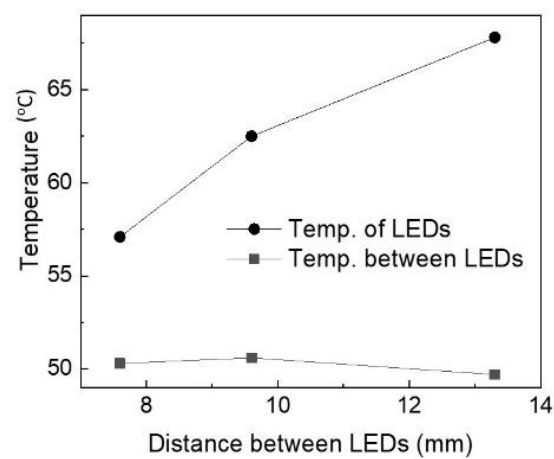

(a)

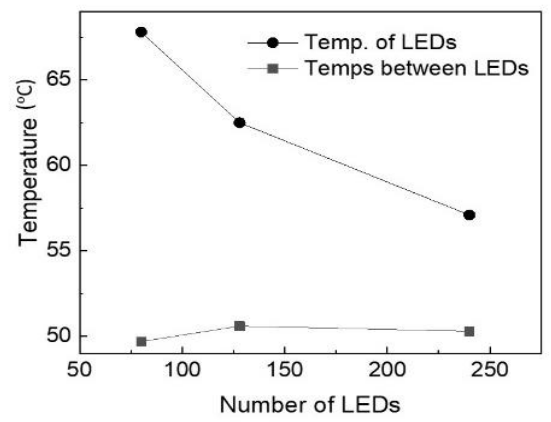

(b)

Fig 6. Temperatures at positions of LEDs and between LEDs as functions of (a) distance between LEDs and (b) the number of LEDs

Published By: 
Fig. 6 shows the variations of temperatures at positions of LEDs p-n junction and between LEDs as functions the distance between LEDs and the number of LEDs. The temperature of LEDs shows the incremental and decremental changes as increasing the distance between LEDs and the number of LEDs, respectively. It can be easily understood because the electrical input power to individual LED and the distance between LEDs depend on the number of LEDs. However, the temperature at position between LEDs showed the different results. It increased when the number of LEDs changed from 80 to 128 as the distance between LEDs changed from $13.3 \mathrm{~mm}$ to $9.6 \mathrm{~mm}$, respectively. After then decreased when the number of LEDs changed from 128 to 240 as the distance between LEDs changed from $9.6 \mathrm{~mm}$ to $7.6 \mathrm{~mm}$, respectively. When the distance is getting close, the heat generated from the LEDs interferes each other. Therefore, the temperature between LEDs can rise. However, the reduction of unit heat flux contributes to reduce the temperature between LEDs although the heat of adjacent LED interferes each other.

\section{CONCLUSION}

The LEDs operating under high temperature led to several disadvantages related to reliability. In order to overcome the disadvantages, Low temperature at the junction should be kept.

Moreover, the number of LEDs on MCPCB should be carefully selected for the optimum operation. The total electrical input power also depends on the property of single LED and MCPCB. And the size of luminaries and the distance between LEDs also should be considered to control the heat dissipation. In this paper, we simulated three arrangements to get the optimum arrangement. The luminous intensity and photo convection efficiency were investigated with respect to various electrical input power. The luminous intensity was increased from 328lm to $1100 \mathrm{~lm}$ when the electrical input power to single LED is adjusted from $1.3 \mathrm{~W}$ to $5.7 \mathrm{~W}$. Whereas, the photo conversion efficiency was decreased from $246.5 \mathrm{~lm} / \mathrm{W}$ to $192.6 \mathrm{~lm} / \mathrm{W}$ when the electrical input power to single LED is increased from 1.3W to 5.7W.

Each LED on PCB need consume less electrical input power with reliability. In order to efficiently design the LED module, three types of the LED module were simulated with different distances between each LED. The distance between each LED was $7.6 \mathrm{~mm}, 9.6 \mathrm{~mm}$, and $13.3 \mathrm{~mm}$, respectively and the number of LEDs are 80,128 , and 240 for $7.6 \mathrm{~mm}, 9.6 \mathrm{~mm}$, and $13.3 \mathrm{~mm}$, respectively. Maximum temperature was $67.8^{\circ} \mathrm{C}, 62.5^{\circ} \mathrm{C}$, and $57.1^{\circ} \mathrm{C}$ for 80,128 , and 240 LEDs, respectively.

We could find the temperature between LEDs were $49.7^{\circ} \mathrm{C}, 50.6^{\circ} \mathrm{C}, 50.3^{\circ} \mathrm{C}$ for 80,128 , and 240 LEDs, respectively. Therefore, the optimum arrangement was assumed to be the module of 128 LEDs.

We showed the right way to find the optimum arrangement when the desired illumination should be designed in terms of the number of LEDs, the total electrical input power. It was to find the inflection point as functions of the distance between LEDs and the number of LEDs on PCB at a given electrical input power conditions. The photo conversion efficiency and the unit heat flux also should be considered.

\section{ACKNOWLEDGMENT}

This article is financially supported by the grant of Hanseo University

\section{REFERENCES}

1. K.C. Yung, H. Liem, H.S. Choy, "Heat transfer analysis of a high-brightness LED array on PCB under different placement configurations", International Communications in Heat and Mass Transfer, Vol. 53, 79-86(2014)

2. E. Juntunen, A. Sitomaniemi, O. Tapaninen, R. Persons, M. Challingsworth, V. Heikkinen, "Thermal performance comparison of. Thick-film insulated aluminum substrates with metal core PCBs for high-power LED modules, IEEE Trans. Compon. Pack. Manuf. Technol. Vol. 2, 1957-1964(2012)

3. B. Pardo, A. Gasse, A. Fargeix, J. Jakovenko, R.J. Werkhoven, X. perpiñà, X. Jordà, M. Vellvehi, T.V. Weelden, P. Bancken, "Thermal resistance investigations on new lead frame-based LED packages and boards, Microelectron. Reliab, Vol. 53, 1084-1094(2013)

4. C.V. Godbold, V.A. Sankaran, J.L. Hudgins, "Thermal analysis of high-power modules, IEEE Trans. Power Electron, Vol.12, 3-11(1997)

5. Min Woo Jeong, Seung Won Jeon, Sang Hun Lee, Yongchan Kim, "Effective heat dissipation and geometric optimization in an LED module with aluminum nitride (AIN) insulation plate", Applied Thermal Engineering, Vol. 76, 212-219(2015)

6. M.Y. Tsai, C.H. Chen, C.S. Kang, "Thermal measurements and analyses of low-cost high-power LED packages and their modules", Microelectronics Reliability, Vol. 52, 845-854(2012)

7. Holman JP. Heat transfer. 8th ed. McGraw-Hill; 2000

8. Sergent J, Krum A. Thermal management handbook for electronic assemblies. McGraw-Hill; 1998.

9. Hsueh-Han Wu, Kuan-Hong Lin, and Shun-Tian Lin, "A study on the heat dissipation of high power multi-chip COB LEDs", Microelectronics Journal, Vol.43, 280-287(2012)

10.Jicheng Zhou, Jinhui Huang, Yunyun Wang, and Zhuang Zhou, “Thermal distribution of multiple LED module", Applied Thermal Engineering, Vol. 93, 122-130(2016)

\section{AUTHORS PROFILE}

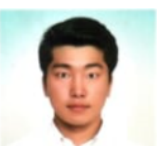

Ju Yong Cho received the B.S. degree in communication engineering and the M.S degree in Optoelectronics from Hanseo University, Seosan, South Korea, in 2014 and 2016, respectively. He is currently Ph.D candidate in Optoelectronics from Hanseo university.

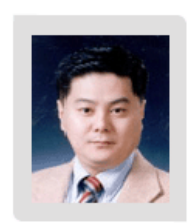

Ho Seob Kim received the Ph.D, in department of Physics, Missouri State University, in 1992. He was a researcher in Thomas J. Watson Research Center from IBM, was a visiting professor in Cambridge University. He is currently a professor in Department of nanoscience, Sunmoon University. His main research interest is electron beam lithography.

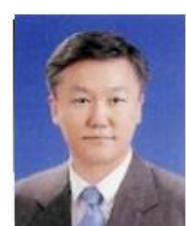

Won Kweon Jang received the B.S., M.S., and the Ph.D., in department of physics from Inha University, Incheon, South Korea, in 1984, 1986, and 1994, respectively. He was a visiting professor in the institute for molecular science, Japan, and he was a Postdoctoral Researcher in the Center for Research and Education in Optics and Lasers(CREOL) from University of Centra Florida. He is currently a Professor in the department of aeronautic electricity, Hanseo University, South Korea. His current research interests are spectroscopy with Fourier transform infrared, micro-chip laser and solid state dye laser. 